\title{
TS-U: Temporal-Spatial Methodology for Application Checking of the Systems in the Ubiquitous Environment
}

\author{
Fran Jarnjak ${ }^{1}$, Jinhyung Kim ${ }^{1}$, Yixin Jing ${ }^{1}$, Hoh Peter In $^{1}$, \\ Dongwon Jeong ${ }^{2}$, and Doo-Kwon Baik ${ }^{1}$ \\ ${ }^{1}$ Dept. of Computer Science and Engineering, Korea University, Seoul, Korea \\ \{fran, koolmania, jing, hoh_in, baikdk\}@korea.ac.kr \\ ${ }^{2}$ Dept. of Informatics \& Statistics, Kunsan National University, Gunsan, Korea \\ djeong@kunsan.ac.kr
}

\begin{abstract}
In the paper a novel methodology, TS-U, is proposed based on existing model checking techniques that were extended to successfully model the systems in a ubiquitous environment by introducing time and space constraints relevant in modeling of some ubiquitous system for its validation. Method proposed requires only slight modification of the existing model checking tools by introducing the notion of a Transition Checking Matrix (TCM) which holds time and space constraints for model's transitions. By applying TS-U methodology, regular CTL formulae can be used without modifications to successfully check the system's model as in the traditional model checking based on the Kripke structure.
\end{abstract}

\section{Introduction}

Nowadays, with rapid advancements in communication technologies and miniaturizations of computational devices many of the current and future systems are, and will, become mobile. Computational and communication ability will be present in many devices from household items, office equipment, apart from the already present wearable devices such as mobile phone, PDA and the like. Thus, a ubiquitous computing environment will become a normal computing environment where various devices communicate in an ad-hoc way, sharing their physical resources and data. When designing applications in such a setting, it is important to check that the application performs as intended due to environment's dynamics, because devices in some area can appear, disappear and request services in a non-predictable random way. Therefore, in ubiquitous environment we should consider both space (room, building, neighborhood, etc.) and time, since different actions can be performed only in particular space at the particular time frame. On the same token, same actions can transition to some other space and continue or stop depending on the time, be it either discrete or in a periodic.

Thus, space and time are interrelated in the ubiquitous environment and should be used in parallel to check some application's correct behavior. In this paper we present a Temporal-Spatial methodology for system checking in the ubiquitous environment called TS-U, which can be used to model some specific ubiquitous system 
(application) and then use the model checking procedures to reason and check for the correct behavior of a modeled system.

This paper is organized as follows. In Section 2 background research is presented where temporal and spatial logic is briefly explained, along with other researches on the temporal-spatial logic. Section 3 introduces the TS-U methodology where it is being applied on the example presented in the Section 4. Section 5 concludes the paper where future work is presented.

\section{Background Research}

Model checking based techniques [1, 2, 3] based on temporal logics [4] are used to express system requirements to verify the design of various systems, hardware or software. Two main paradigms are symbolic and explicit-state representations for the system states implemented in SMV [5] and SPIN [6]. In CTL model checking, the system is modeled as a Kripke structure and its desired properties to be verified are represented in CTL formulae. Kripke structure $K=(S, T, L)$ is a labeled finite state machine, where $S$ is a set of states, $T$ is the transition relation which is required to be total and $L$ is a labeling function, labeling transitions with atomic propositions $A$ from one state to another. CTL formulas are concatenation of atomic propositions and basic operators such as " " standing for "not", "V" standing for "or" and characters "E", "X", "G" and "U" standing for "there exists", "next", "global" and "until". In model checking using CTL, one would design a Kripke structure representing the system and label the transitions. In each state certain propositions would hold or not. Then, the system would be checked using a CTL formula in order to determine its validity. For example, if one would model a microwave-oven system, where the door can be opened or not and the power can be on or off, one could use the CTL operators to check that there is no case when the door is opened and power is on where microwave oven is radiating causing a safety hazard.

However, model checking using Kripke structure is not well suitable for continuously changing influences, but is more suited for discrete changes. For example, one could not model easily some variable whose influence depends on some interval that can be used to verify the validity of the system. For example, suppose a variable $x$ can have a value from between 0 and 10, and depending on its value a system would have different properties that are valid or not. In such a case, a designer would have to introduce 10 different states to correctly model the system using a Kripke structure or a designer would have to select some degree of granularity, say introducing a state for which $x$ value is between $0-5$ and another for values between 610 to represent some interested states of the system; however the model may not be exact representation of the real world. Therefore, large number of states is required to model a system correctly causing a state explosion problem.

In this paper, proposed technique does not require additional states since restrictions are imposed on the transitions and not on states, thus existing tools can be used directly with minor modifications and variables based on intervals (i.e. time) can be successfully modeled.

In [7] a spatial logic based on regions and connections is presented where interval logic for reasoning about space is presented. Authors define a basic primitive relation 
$C(x, y)$ that reads "x connects with y" defined on regions and it holds whenever regions $\mathrm{x}$ and $\mathrm{y}$ share a common point. In terms of space, a subsumption hierarchy is defined on how two spaces can be related to each others consisting of 8 different relations: PO (partial overlap), TPP (tangential proper part), NTPP (non-tangential proper part), = (equal), $\mathrm{TPP}^{-1}$ (inverse of TPP), $\mathrm{NTPP}^{-1}$ (inverse of NTTP), EC (externally connected) and DC (disconnected). Thus, the logic is more commonly known as RCC-8. However, the RCC-8 was developed to be universal and general, whereas it can be much more simplified when the ubiquitous environment is considered, as in this paper. For example, in the RCC-8 logic there is a clear distinction between TPP $(\mathrm{a}, \mathrm{b})$ where $\mathrm{a}$ is enclosed inside $\mathrm{b}$ touching $\mathrm{b}$ in one point (tangential) and NTPP $(a, b)$ where $a$ is enclosed in $b$ but not touching its boundary. However, if we suppose a classroom is inside a building, where both classroom and building have some shared and unique properties that have to be verified, we would not be concerned if a classroom is at the buildings corner (TPP) or somewhere not touching external building's walls (NTPP). Thus, in this paper more simplified spatial relationships are addressed without the loss of generality when ubiquitous environment is considered.

In [8] a modal logic for mobile ambients is proposed whose purpose is to study mobility defined as a change of spatial configurations over time. Thus, logic proposed talks about space and time and has the Ambient Calculus as a model. Properties in such logic can hold at particular locations, i.e. space, and spatial configurations evolve over time as a consequence of the activities of some processes. Logic is mostly developed to automate the checking of mobile code in mobile environments for security purposes. However authors, when dealing with time and space issue, are concerned about the "sometime" and "somewhere" constraints. That is, the logic proposed is not concerned with specific time intervals or space, which is required in a ubiquitous environment. In other words, having "sometime" and "somewhere" constraints does not allow for a more complete and explicit model to be created whose properties can be checked. In this paper, specific time intervals and spatial relations are given, thus making modeling more expressive and correct in representing real world applications.

\section{TS-U Methodology}

TS-U methodology concerns time and space and applies it to the already existing model checking procedures as described in the previous section. Thus, we will deal with time and space issue separately in the following subsections and then present a method to combine them and apply them to the model checking useful in the ubiquitous environment.

\subsection{Time}

Considering time and applying it to the ubiquitous environment we can consider four possible cases with or without repetition. In this paper, we adopt a 24-hour time system for clarity. The time can be one discrete time point, it can signify the time before or after some time point or it can specify a time period from one time point to 
another time point and time can be represented as infinity, which means some event can occur at any time. Lastly, some time points can repeat themselves over a period of time as well. To clarify, let's consider the following examples:

- “A meeting at 10 , Tuesday, March $8^{\text {th }}, 2005$ " is considered to be a discrete non-repetitive time point.

- "A meeting every Monday at 14" is considered a repetitive time point which repeats every 7 days.

- "Watching TV after 20 on Tuesday, March $15^{\text {th }}, 2005$ " is considered a nonrepetitive time point that occurs after 20 hours on March $15^{\text {th }}, 2005$.

- "A presentation from 15-16, on Tuesday, March $15^{\text {th }}, 2005$ " is a time period which is a non-repetitive time period occurring only on March $15^{\text {th }}, 2005$ from 15 to16 hours.

- "A class from 12-13 every Friday" is a repetitive time-period occurring from 12-13 hours every Friday.

To reduce the number of variables to represent time, we can adopt a time system like UNIX system time [9] which specifies the number of seconds elapsed since the beginning of the epoch (1/1/1970). Thus, instead of dealing with hours, minutes, seconds, days, months and years separately, we obtain one unique number for some specific time.

Adopting such a time representation, we can define possible time configurations more formally as in the Table 1 below:

Table 1. Possible time configurations

\begin{tabular}{cl}
\hline Time Category & \multicolumn{1}{c}{ Description } \\
\hline$t+\Delta r$ & Infinite time \\
\hline$<(t+\Delta r)$ & $\begin{array}{l}\text { Discrete time point occurring once at } \\
\text { time } t \text { if } \Delta r=0 . \text { In case of repetition, } \Delta r \\
\text { represents the period of next occurrence } \\
\text { from the starting time point } t \text {. }\end{array}$ \\
\hline$>(t+\Delta r)$ & Represents time before some discrete \\
& time point $t$ when $\Delta r=0$. In case of \\
& repetition, $\Delta r$ represents the period of \\
& the next occurrence of such time \\
& specification. \\
\hline & Represents time after some discrete time \\
& point $t$ when $\Delta r=0$. In case of repetition, \\
& $\Delta r$ represents the period of the next \\
& occurrence of such time specification. \\
\hline & Represents a time period starting at a \\
& discrete time point $t$ with duration of $d$. \\
In case of repetition, $\Delta r$ represents the \\
period of the next occurrence of such \\
time specification.
\end{tabular}


Since we are concerned about if certain transition in the Kripke structure can occur or not at some specific time compared with the allowed interval, it is not necessary to consider the relationships between intervals. That is, it is not relevant to check if some interval happens before or after some other time interval, since main concern is to check if the current system time matches some specified time interval or not to allow or not allow the transition to occur.

\subsection{Space}

Space is defined as a closed, bounded area where an activity can occur. Usually, as explained in the background section above, there are several possible cases how space can be configured, for example two space regions can touch each other tangentially, overlap or one can be enclosed in another, etc. However, in case of ubiquitous environment we are considering the space configuration at a specific time point. Thus, not all cases presented in [7] need to be considered. For example, in case two spaces touch each other tangentially, a person can be only in one space at the time if they are adjunct to each other (e.g. rooms next to each other). In case two spaces overlap or one space is contained in another and a person is in such a place, then a person is present in both places at the same time (e.g. room in a house; a person is both in the room and in the house at the same time), or in case person is outside the contained space (e.g. front door of the house). In case two spaces partially overlap each other, person can be in one space or the other, or in the overlapping area (e.g. house has a yard that has a terrace, however terrace is built on the yard; thus, person can be either in the house, on the yard or on the terrace which is both a house and a yard). Further, some space can have more actions that are allowed in it than the other. For example, a professor in a campus building has more resources and rights in his private office than on the hallway of the same building.

Therefore, a taxonomy of spaces as in [7] should be considered fully, and in the proposed approach the children would inherit the rules of what activities can occur (at a specific time) from their parents and can add additional rules to itself. An example of such taxonomy is depict in the Figure 1 below:

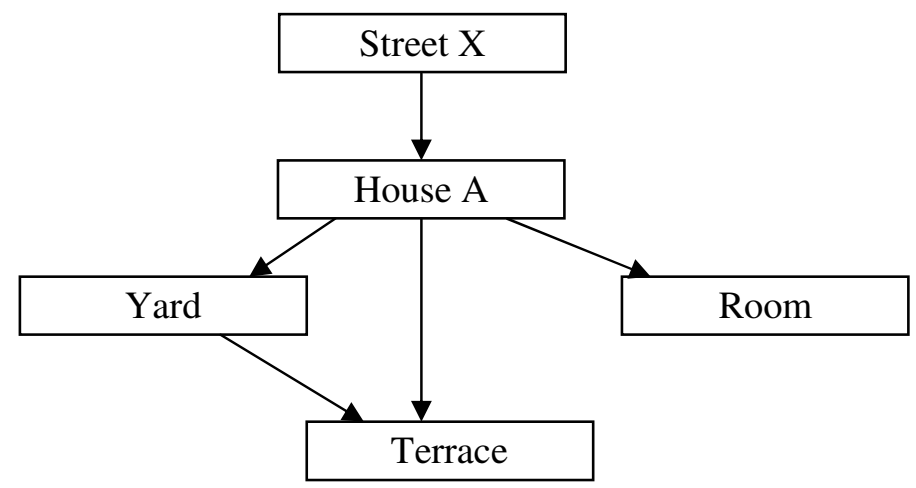

Fig. 1. An example taxonomy of space configurations and their relationships 
Therefore, rules of the "Street $X$ " that can occur there are inherited by the "House $A$ ", since "House A" belongs to the "Street $X$ ". Rules of both "Street $X$ " and "House $A$ " are inherited by both the "Yard" and the "Room" since they both belong to the "House A" and "Street $X$ ", however they can add their own rules, if there such a need. Lastly, the "Terrace" inherits rules of both the "Yard" and the "House A" (and "Street X"), since it is both in a "Yard" and a part of the "House A".

More formally we can define an inherit operator $\leftarrow$, for example $A \leftarrow X$ that specifies that some space inherits from another space. That is, rules along with their specified time-frames are inherited from space $X$ and now belong to the space $A$, since space $A$ subsumes $X$.

As mentioned earlier, each space can have a rule set called RS. For the rule specification, we adopt propositional logic where the following Boolean operators are allowed: " $\neg$ " for NOT, " $\wedge$ " for AND and " $\vee$ " for OR. We write $A\left[R S_{A}\right]$ meaning that a space $A$ has a rule set $R S_{A}$. In case of inheritance, inherited rules from the parents are joined in the set of the children. For example, $A\left[R S_{A} \cup R S_{X}\right] \leftarrow X\left[R S_{X}\right]$ means, that space $X$ has a rule set $R S_{X}$ and space $A$ has both its own rule set $R S_{A}$ and inherited rule set $R S_{X}$. Rules $R$ in a set form a tuple of the propositions $P_{i}$ and time specifications $T_{i}$, which is written as $\left(\mathrm{P}_{\mathrm{i}}, \mathrm{T}_{\mathrm{i}}\right)$. Thus, $R S_{X}$ for the "Street $X$ " can have an element such as (walk, $\infty$ ) where $\mathrm{P}_{1}=$ walk and $\mathrm{T}_{1}=\infty$, meaning that someone is allowed to walk on the street at any time. However, sometimes inherited rules violate the policies that could be present in the children's space, in which case they can be omitted from inheritance by the system modeler.

Therefore, considering the case as in Figure 1, possible rules, with inheritance displayed, could be as follows:

(1) $X[($ walk, $\infty)]$

(2) A[(sleep, $>100+500),(\neg$ smoke, $\infty),($ walk, $\infty)] \leftarrow X[($ walk, $\infty)]$

(3) $\operatorname{Yard}[($ smoke, $\infty),($ walk, $\infty)] \leftarrow A[($ sleep, $>100+500), \quad(\neg$ smoke, $\infty),($ walk, $\infty)] \leftarrow X[($ walk, $\infty)]$

In the above rule (1), a person is allowed to walk on the "Street $X$ " anytime. Rule (2) says a person in the "House A" can sleep after time 100 with the repetition period of 500 (we omitted real time since epoch to keep the numbers small), he/she can never smoke and can always walk. Rule (3) states a person can always smoke and walk in the "Yard", sleeping and not smoking rule is not inherited from the parent "House A" (suppose sleeping is dangerous and smoking is allowed so the nonsmoking rule is overridden).

\subsection{Transition Checking Matrix (TCM)}

Once the treatment of time and space has been defined separately, for model checking methodology in the ubiquitous environment time and space has to be treated simultaneously, because of their interrelated relationship. In other words, some action in the ubiquitous application can be performed if and only if both time and space agree according to the rules. Thus, to check if some action is allowed or not we introduce the Transition Checking Matrix (TCM). TCM contains the action itself, space set and rule set, similar to the rules in the previous subsection. When model checking the application, TCM is consulted and if both space and time agree, an 
action can be performed. TCM has the following structure, where example rows have been inserted from the previous subsection for clarity:

Table 2. Example of the TCM

\begin{tabular}{ccc}
\hline Action & Space & Time \\
\hline walk & X & $\infty$ \\
\hline sleep & A & $>100+500$ \\
\hline$\neg$ smoke & A & $\infty$ \\
\hline smoke & Yard & $\infty$ \\
\hline
\end{tabular}

If some space has multiple actions, multiple rows are inserted with the action name and time constraint, as is the case for space $\mathrm{A}$ in the Table 2 above.

\subsection{Model Checking Using TCM}

Existing model checking explained in Section 2 of this paper, uses Kripke structure to model and check the system for correct behavior using rules specified by the CTL logic. In this paper TS-U is proposed that extend already existing model checking methodology, by applying TCM matrix for applying additional constraints on the transition labels. In other words, a transition in Kripke structure can occur from one state to another if both space and time match. Transition labels (actions) are put in the first column of the TCM matrix. Space and time are dealt as external variables that provide environment input to the ubiquitous system modeled with the Kripke structure. Conceptually, the system can be looked as depict in Figure 2 below:

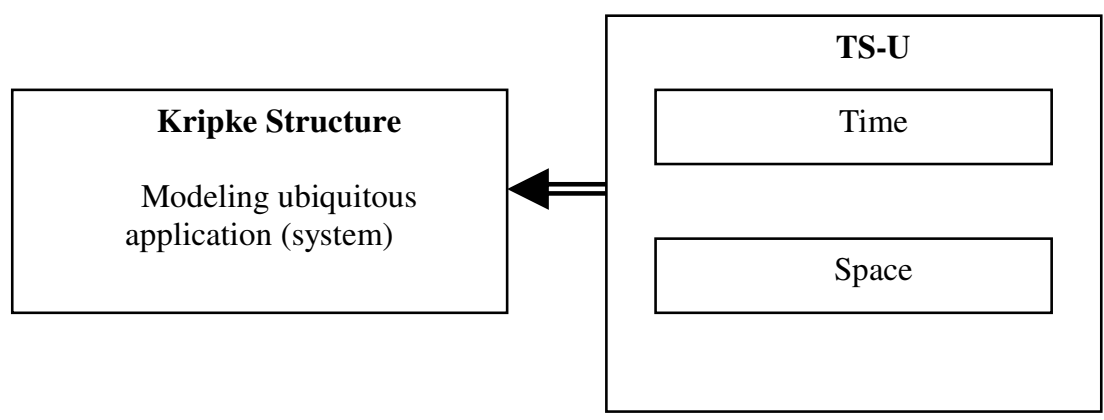

Fig. 2. TS-U conceptual view

In Figure 2 above, on the left is the regular existing model using the Kripke Structure that models some ubiquitous system. On the right is the proposed TS-U methodology that provides external input concerning time and space to the Kripke structure. Model checker uses CTL to check the system. However, each time a transition should be made TS-U is consulted where transition is checked against TCM concerning space and time and thus a transition is either allowed, if both time and 
space constraints are matched for such a transition, or not allowed if either time or space or both are not matched.

It is important to note that during model checking, a time frame is specified in order to prevent infinite execution (say, model checker is in a certain state and no transition can occur since space/time constraint is never met). For example, if we create a model of some ubiquitous application in a university environment, say "smart campus" studied in [10], we may be interested in checking that the system operates as expected in a one-week time frame, since during the semester events are usually repetitive. Thus, granularity of time should be a variable parameter as a part of model checker program's input. On the same token, we assume location is known a priori every time verification is performed against TCM. Thus, when the time frame period finished model checking stops and CTL formula results are presented. If for some CTL formula model checker did not finish its evaluation and the time frame period finished, such formula is evaluated to false since for the interested time period its truth is false. Indeed, for the same formula if the period is longer (say, instead of one week, two weeks) the same formula may be evaluated to true, if some action has repetition granularity of two weeks. However, as an input to the model checker when time was specified as "one week", the formula would be evaluated to false.

\section{Example Application Scenario}

In order to show the application scenario of proposed TS-U methodology, let's consider a university environment, consisting of a professor as an entity performing ubiquitous actions, his office, a lecture room and a meeting room. A professor can perform various activities in his office, such as use email, phone or fax machine. He has a lecture every Monday from 14:00-15:00 where he can use a projector and whiteboard and a meeting every Tuesday from 10:00-11:00 where he can use a notebook computer. Activities performed are: "give lecture", "return to office" and "go for a meeting". Thus, the following Kripke structure can be devised as presented in the Figure 3 below:

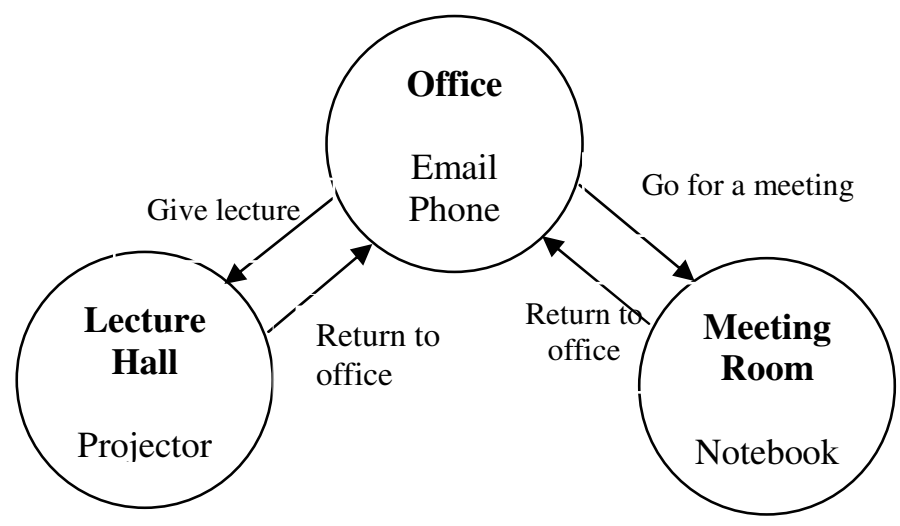

Fig. 3. Kripke structure of an example scenario 
In Figure 3 above, regular Kripke structure is devised, where existing model checking techniques based on CTL can be used. However, in the ubiquitous environment, constraints on transitions ("give lecture", "return to office" and "go for a meeting") have to be made, since many of the resources are limited and can only be used by persons at a specific time and place. For example, in the scenario above, a professor can give lecture only from 14:00-15:00 on Monday where his role is of a lecturer, but he can attend some other lecture, say workshop, in the same lecture hall given by others, so he can not use a projector and a whiteboard at that time in that place. Thus, constraints based on proposed TS-U methodology can be as follows:

Table 3. TCM of TS-U for the example scenario

\begin{tabular}{lll}
\hline Action & Space & Time \\
\hline Give Lecture & Lecture Hall & $(100+3600,604800)$ \\
\hline Return to office & Office & $\infty$ \\
\hline Go for a meeting & Meeting room & $(300+3600,604800)$ \\
\hline
\end{tabular}

In the table above, a professor can transition from an office space to the lecture hall space at time 100 (suppose time 100 is Monday at 14:00) and use its resources for 1 hour with a repetition of 7 days. Naturally, he can return to his own office at any given time, even during the lecture or meeting, etc. Lastly, he can transition from his office to the meeting room and use its resources at a time 300 (suppose time 300 is Tuesday at 10:00) where he can use the resources for 1 hour with a repetition of 7 days. It should be noted, that TCM does not contain rows for a professor transitioning to the lecture hall in case of a workshop mentioned earlier, since no resources would be needed when transition occurs, thus validity of the model should not be in question.

Once the above model has been created using the proposed approach, existing CTL formulae can be used to verify the properties of interest without modifications, like they would be used if the model was created using a regular Kripke structure as in the previous approaches discussed in Section 2 of this paper.

\section{Conclusion}

In this paper an TS-U methodology was proposed that extends existing model checking techniques based on CTL logic, that combines both space and time which are additional constraints on the model being checked, which is an important factor in the ubiquitous environment. TS-U utilized Transition Checking Matrix (TCM) to verify if some transition can occur or not. For future work, we would like to extend existing model checking tool, SMV [5], to implement proposed TS-U methodology to obtain more concrete results and also to extend TS-U to another level where actions can be tied to individuals, thus same model in terms of the Kripke structure can be reused. 


\section{Acknowledgement}

Doo-Kwon Baik and Hoh Peter In are the co-corresponding authors.

\section{References}

[1] E. Clarke, E. Emerson, and A. Sistla. Automatic verification of finite-state concurrent systems using temporal logic specifications. ACM Transactions on Programming Languages and Systems, 8(2):244-263, 1986.

[2] J. Quielle and J. Sifakis. Specification and verification of concurrent systems in CESAR. In Proceedings of the Fifth International Symposium in Programming, LNCS 137:337$351,1981$.

[3] Edmund M. Clarke, Jr., Orna Grumberg and Doron A. Peled. Model Checking. The MIT Press, Cambridge, Massachusetts, 1999.

[4] A. Pnueli. The temporal logic of programs. In Proceedings of the $18^{\text {th }}$ Symposium on Foundations of Computer Science, pages 46-57, 1977.

[5] K. McMillan. Symbolic model checking: an approach to the state explosion problem. PhD thesis, School of Computer Science, Carnegie Mellon University, 1992.

[6] E. Clarke, E. Emerson. Design and synthesis of synchronization skeletons using branching-time temporal logic. In Proceedings of the Workshop on Logic of Programs, LNCS 131:52-71, 1981.

[7] D.A. Randell, Z.Cui, A.G.Cohn. A Spatial Logic based on Regions and Connection. In Proceedings of the $3^{\text {rd }}$ International Conference on Knowledge Representation and Reasoning, Morgan Kaufman, 1992.

[8] L. Cardelli, A.D. Gordon. Anytime, Anywhere Modal Logics for Mobile Ambients. In Proceedings of POPL Conference, 2000.

[9] Bell Labs. Unix Programmer's Manual. First Edition, 1971. http://cm.bell-labs.com/cm/cs/who/dmr/1stEdman.html

[10] L. Barkhuus, P. Dourish. Everyday Encounters with Context-Aware Computing in a Campus Environment. In Proceedings of UbiComp 2004 conference on Ubiquitous Computing, 2004. 